Check for updates

Cite this: RSC Adv., 2018, 8, 3453

\title{
An ultra-compact blackbody using electrophoretic deposited carbon nanotube films
}

\author{
Albert Lin, (D)* Chien-Chih Yang, Parag Parashar, Chien-Yung Lin, Ding Rung Jian, \\ Wei-Ming Huang, Yi-Wen Huang, Sze Ming Fu, Yan Kai Zhong \\ and Tseung Yuen Tseng*
}

Carbon nanotubes (CNTs) possesses decent optical properties and thus can be considered as a candidate for perfect absorbers due to their close-to-air refractive index and minimal extinction. However, weak absorption in porous materials, due to the low extinction coefficients, requires an inevitably thick absorption layer $(\sim 100 \mu \mathrm{m})$ for the perfect opaque absorbers. Thus, the requirement of large thicknesses of CNTs prohibits them from being used as miniaturized integrated photonic devices. Here, we propose an electrophoretic deposited (EPD) CNT resonant cavity structure on tantalum (Ta) to enhance optical absorption. Efficient random light scattering along with the resonant cavity structure using $\mathrm{Ti} / \mathrm{SiO}_{2}$ stacking enhances the absorption in our proposed EPD-CNT film while maintaining the total device thickness to $<1 \mu \mathrm{m}$. The experiment results reveal that the absorption band covers the entire UV-VIS-NIR spectrum $(\lambda=0.3-2.6 \mu \mathrm{m})$, using resonant-cavity EPD-CNT design. The EPD deposition process is done at relatively low temperature $<120{ }^{\circ} \mathrm{C}$. We believe that this proposal is very promising for sensing, antenna, and thermophotovoltaics (TPV), in terms of bandwidth, compactness and cost.

Received 4th November 2017 Accepted 10th January 2018

DOI: $10.1039 / c 7 r a 12113 j$

rsc.li/rsc-advances incoming photons due to the high extinction coefficients of the metallic materials. However, the problem associated with the metallic MPAs is that the coupling of the incoming photons with the metallic layers can be difficult because of the significant refractive index difference between the metals and the ambience. Owing to this reason different schemes have been proposed in recent years to increase the photon coupling and photon confinement inside the metallic or metal-dielectric nanostructures, in order to achieve a perfect blackbody absorption over a broad spectral range. These include adiabatic coupling, ${ }^{14-16}$ plasmonics,${ }^{10}$ hyperbolic dispersion, ${ }^{14}$ and field penetration ${ }^{17}$ phenomenon. Nonetheless, extensive use of lithography and etching processes also impose an extra cost and time inefficiency in most of these structures.

In contrast to metal-dielectric nano-photonic perfect absorbers, porous materials present another route for high absorption. Porous materials have close-to-unity real refractive index, and therefore, the in-coupling of the photons into the porous materials is not an issue due to their close-to-air refractive indices. As far as porous materials are concerned, CNTs has a higher melting point, higher mechanical and tensile strength than other noteworthy porous metal oxides such as NiO, $\mathrm{Fe}_{2} \mathrm{O}_{3}, \mathrm{Co}_{3} \mathrm{O}_{4}, \mathrm{Mn}_{2} \mathrm{O}_{3}, \mathrm{NiFe}_{2} \mathrm{O}_{4}$, and aluminum oxide, ${ }^{18}$ thereby, suitable for high temperature and harsh environment applications, such as thermophotovoltaics (TPV). Besides, efforts are being made in recent years to grow CNTs at a relatively low temperature $\left(150{ }^{\circ} \mathrm{C}\right),{ }^{19}$ as compared to high temperature CVD process, to lower the cost of CNT raw materials. 
Inherent low extinction coefficient of CNT and other porous materials leads to a weak absorption and thus a large device thickness is required to achieve in the optical regime. Due to this constraint, CNTs are thus excluded from being categorized as an effective MPAs. ${ }^{6,20}$ Instead, CNTs are more often referred to as opaque materials rather than a photonic device. To achieve the high-absorption property, the required thickness of carbon nanotube absorber ${ }^{21-26}$ is usually about tens to hundreds of micrometers as discussed previously. Among these advancements, a $70 \mu \mathrm{m}$ thick CNT grown on glass substrate with $1 \mathrm{~nm}$ Fe seed layer using $775{ }^{\circ} \mathrm{C}$ CVD is shown to achieve perfect blackbody. ${ }^{22} \mathrm{~A}$ forest of vertically aligned single-walled carbon nanotubes (SWNTs) ${ }^{23}$ behaves most similarly to a black body for the $\mathrm{UV}(200 \mathrm{~nm})$ to far infrared $(200 \mu \mathrm{m})$ regime. Besides, dark materials made by a low-density nanotube array ${ }^{26}$ also have been engineered to have an extremely low index of refraction together with the nanoscale surface roughness of the arrays to induce a nearly-perfect optical absorption material. The fact remains that a large thickness is required is a common problem when perfect absorbers are realized using highly porous materials to match the impedance of air. Due to the large thickness restraint of CNT absorbers, it can be hard to integrate the CNT absorbers with other nanoscale devices. Therefore, thickness minimization of CNT absorbers while maintaining their high absorption property is the key issue for the integration of CNTs with other optical devices. The recent work by N. Selvakumar et al. ${ }^{27}$ reports the $800{ }^{\circ} \mathrm{C}$ chemical vapor deposition (CVD) CNT on stainless steel substrate using tandem absorption of CNT and metallic substrate that reduced CNT thickness requirement to several micrometers. The decent absorption property achieved here ${ }^{27}$ with a reduced thickness is promising. But the extensive use of high processing temperature $\sim 800^{\circ} \mathrm{C}$ prevents the use of flexible substrates along with the possible process integration with many low-temperature materials. Here, in the work proposed we use low-temperature $<120{ }^{\circ} \mathrm{C}$ electrophoretic deposition (EPD) to realize ultra-compact $\mathrm{CNT}$ absorbers with the aid of broadband resonant cavity design. EPD process is an economical and versatile processing technique for the carbon nanotubes (CNTs).$^{28}$ Furthermore, no lithography and etching processes are required in our structure, thereby, adding cost and time efficiency to our device. The miniaturized dimension is $<1 \mu \mathrm{m}$ with broadband absorption over $\lambda=0.3 \mu \mathrm{m}$ to $\lambda=2.6 \mu \mathrm{m}$.

Specifically, we present the process of EPD for fabricating carbon nanotubes, the UV-VIS-NIR and Fourier transform infrared spectroscopy (FTIR) measurement results, and the angular absorption measurement results. Also, we investigate the dependence of broadband absorption on the EPD CNT thickness. Finally, the ultimate purpose is to achieve reducedthickness CNT absorbers with the aid of an optical broadband resonant cavity and the random light scattering within the EPD CNT films. With this low temperature processing together with the resonant cavity, our structure is very efficient in terms of bandwidth, compactness, and the integrability with other photonic devices.

\section{Simulation}

Simulation results have been presented in this section. The simulation software we used is Rsoft DiffractMOD ${ }^{\mathrm{TM}}$ and the absorption calculation is based on the rigorously coupled wave analysis (RCWA).

The material parameters including refractive indices and extinction coefficients have been taken from Rsoft ${ }^{\mathrm{TM}}$ database, ${ }^{29}$ and the CNT refractive index is from a literature. ${ }^{22}$ Fig. 1 shows our proposed structure. The structure is a combination of CNTs and a resonant cavity consisting of a bottom Ta layer and alternating $\mathrm{Ti} / \mathrm{SiO}_{2}$ layers. In principle, if we want to achieve full absorption of light, the thickness of CNTs must be tens to hundreds of micrometers due to their porous nature and the corresponding low absorption coefficients. Therefore, with the integration of resonant cavity, we try to reduce the thickness of CNT perfect absorbers. The thickness of CNTs in the simulation (a)

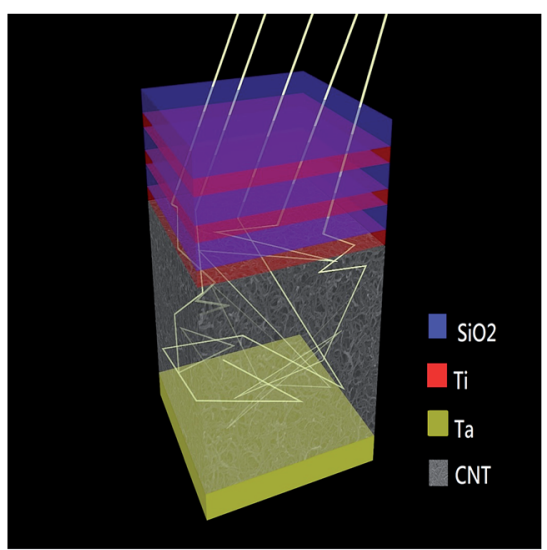

(b)

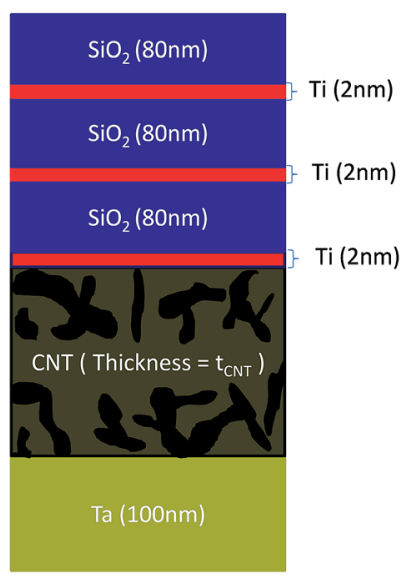

Fig. 1 (a) The three-dimensional (3D) illustration of the cavity resonant EPD-CNT blackbody. (b) The geometry parameters of EPD-CNTs with a cavity. The resonant cavity is composed of 3 pairs of $2 \mathrm{~nm} \mathrm{Ti}$ and $80 \mathrm{~nm} \mathrm{SiO}$ alternating layers. The high-temperature refractory metals Ta and Ti are employed. 
(a)

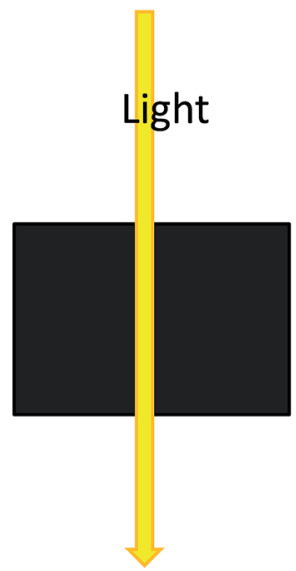

(b)

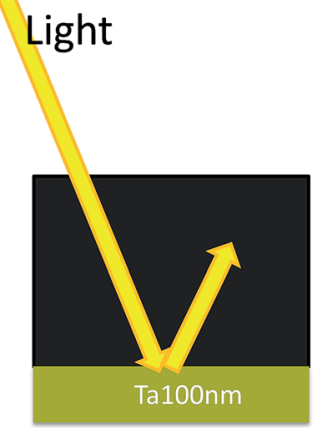

(c)

Light

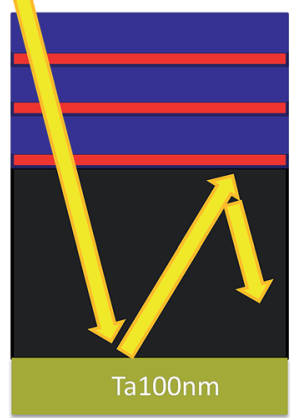

Fig. 2 (a) The light path in blanket CNTs, (b) CNTs with a backside Ta reflection layer, and (c) CNTs with a resonant cavity.

is $700 \mathrm{~nm}$, and the cavity is composed of three pairs of alternating $2 \mathrm{~nm}$ Ti and $80 \mathrm{~nm} \mathrm{SiO}_{2}$ thin films. The $100 \mathrm{~nm}$ Ta on the bottom serves as the bottom reflection layer to eliminate transmittance and also serves as the electrode during EPD.

Fig. 2 illustrates the light paths for the standalone CNT blanket and the CNTs with a resonant cavity. A significant portion of photons can penetrate standalone CNTs while photons can be fully absorbed by CNTs due to the confinement achieved by the resonant cavity. The simulation results in Fig. 3 demonstrate this phenomenon. The absorption of CNTs with a cavity is much higher than the blanket of standalone CNTs over the entire broadband wavelength range from $\lambda=300 \mathrm{~nm}$ to $\lambda=2600 \mathrm{~nm}$. The oscillations in the absorption are due to the fact that the resonance phenomenon is very pronounced in the cavity structure. Fig. 3(b) further shows the omnidirectional property of the structure where the absorption of $60^{\circ}$ incidence at TE and TM polarizations is not decreased over the same broad spectral range $\lambda=300 \mathrm{~nm}$ to $\lambda=2600 \mathrm{~nm}$ as compared to the normal incidence. It should be noted that the refractive index of the CNTs is assumed to be $1.02+0.04 i .^{22}$ Thus, it is a porous and weak absorption material that has been employed in the simulations. The absorption enhancement due to the random light scattering in EPD-CNTs has not been reflected in this section since the highly random, highly entangled EPDCNT structure is not in fact computationally manageable. As a result, the experimental absorption can be even superior in terms of absorption perfection and bandwidth, compared to the simulation results. The field profiles are presented in Fig. 4. It

\section{(a)}

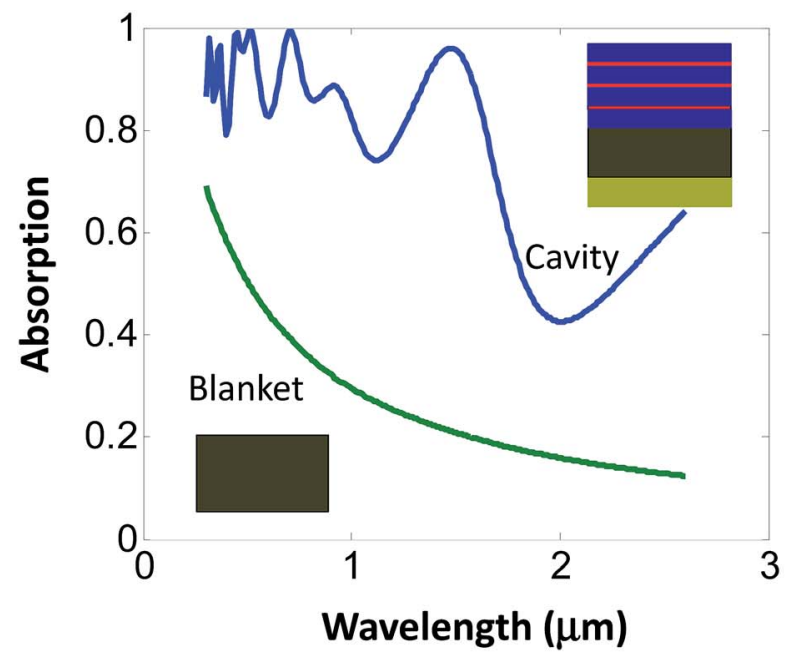

(b)

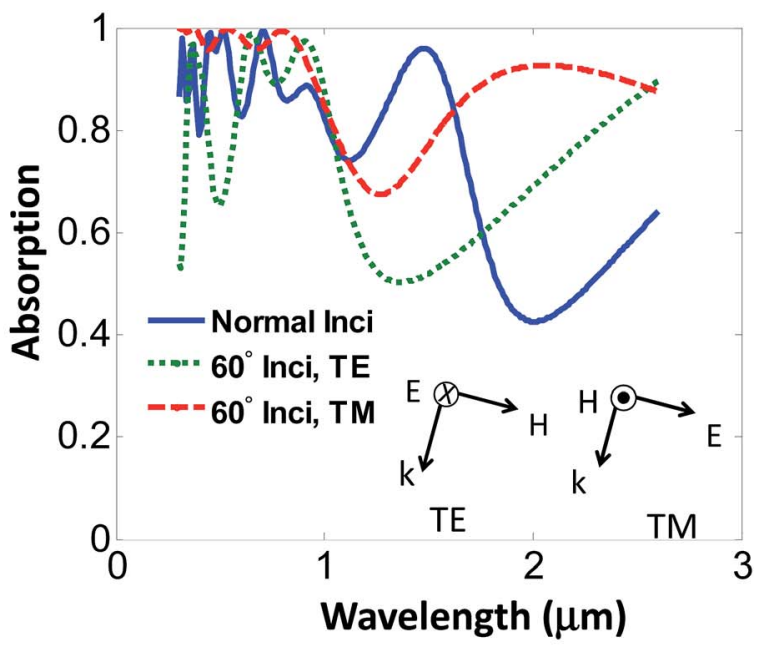

Fig. 3 (a) The normal incidence spectral absorption for blanket CNTs and the CNTs with cavity design. (b) The angular spectral absorption for the CNTs with cavity design. The spectral absorption for TE and TM polarizations with $60^{\circ}$ incidence angle is shown. $t_{C N T}=700 \mathrm{~nm}$ for the blanket CNT film and the cavity-resonant type CNT absorber. 3.5-pair $80 \mathrm{~nm}-\mathrm{SiO}_{2} / 2 \mathrm{~nm}$-Ti and a $100 \mathrm{~nm}$ Ta bottom layer are used to form the resonant cavity. 

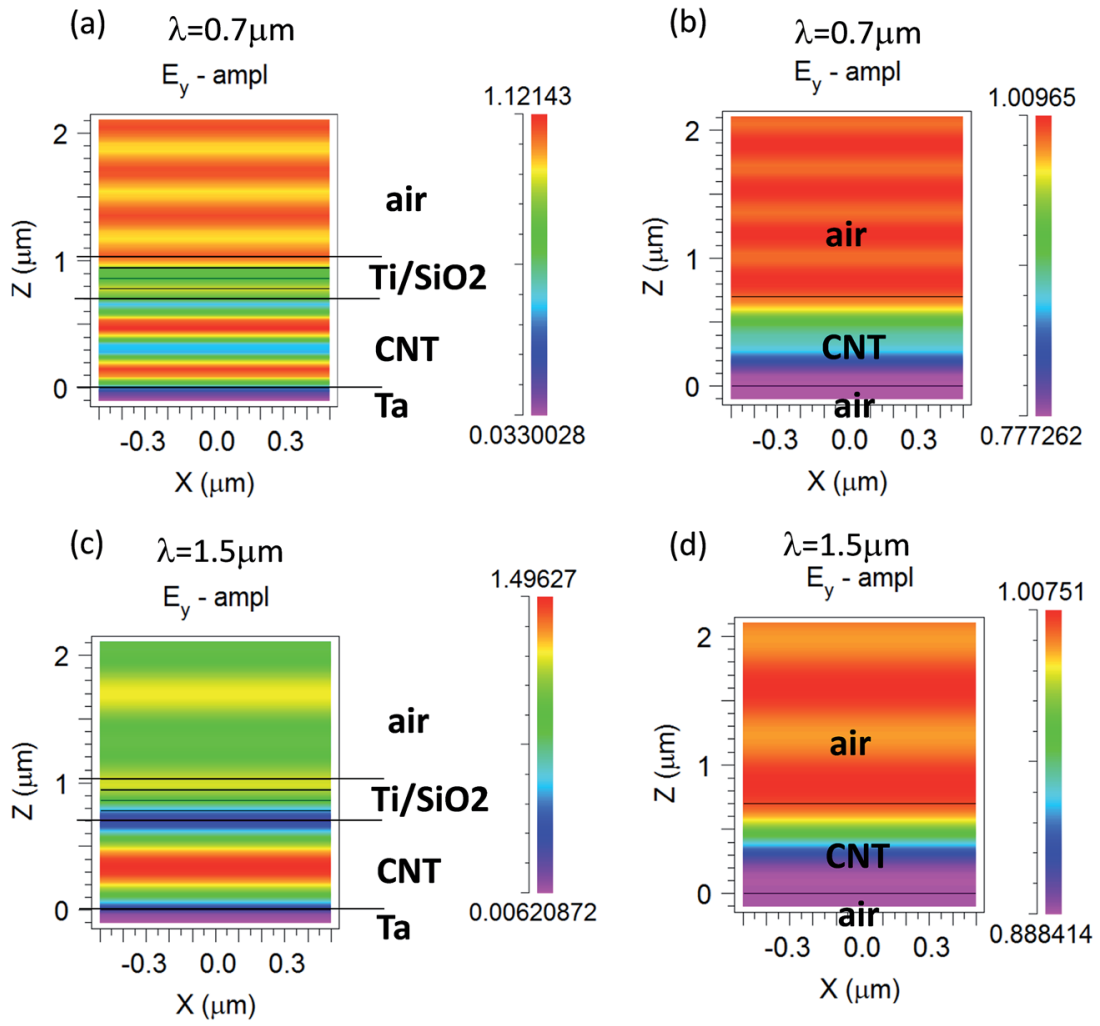

Fig. 4 The time-harmonic steady-state $E_{\mathrm{y}}$ amplitude for (a) CNTs with a cavity, at $\lambda=0.7 \mu \mathrm{m}$ (b) blanket CNTs, at $\lambda=0.7 \mu \mathrm{m}$ (c) CNTs with a cavity, at $\lambda=1.5 \mu \mathrm{m}$ (d) blanket CNTs, at $\lambda=1.5 \mu \mathrm{m}$. $t_{\mathrm{CNT}}=700 \mathrm{~nm}$ is assumed for the blanket CNT film and the cavity-resonant type CNT absorber. 3.5-pair $80 \mathrm{~nm}-\mathrm{SiO}_{2} / 2 \mathrm{~nm}-\mathrm{Ti}$ and a $100 \mathrm{~nm}$ Ta bottom layer are used to form the resonant cavity.

can be clearly seen that the electromagnetic field is effectively confined in CNTs with the resonant cavity design. Without the $\mathrm{Ta}$ and $\mathrm{Ti} / \mathrm{SiO}_{2}$ resonant cavity, the electromagnetic field directly penetrates the standalone blanket of CNTs since $700 \mathrm{~nm}$ CNTs are not thick enough to fully absorb the incident fields, as evident from Fig. 4(b) and (d). As a result, with the integration of the resonant cavity to the structure, the thickness of CNT absorbers can be minimized to several hundred nanometers. It will be clear later in the Experimental section that with the aid of random light scattering in EPD-CNTs, the measured absorption of a thin EPD-CNT device can be increased further from the simulated values.

\section{Experiment}

In this section, we present the experimental results for the proposed cavity-type EPD-CNT structure. Since the thickness of CNT films has to be confirmed, we measure the thickness dimensions by scanning electron microscope (SEM). Fig. 5(a) is the SEM micrographs of the films, and Fig. 5(b) is the Raman spectroscopy. Various thicknesses of CNT-EPD films are deposited on Ta substrate in this work. Fig. 5(a) also shows the zoom-in micrograph of the CNTs cross-section to show the randomly distributed nature of EPD-CNTs. In addition, random distribution of CNTs is also evident in the top view, therefore, supports our discussion that the enhanced broadband absorption is in part due to the random light scattering within EPD-
CNTs. In the Raman spectroscopy, we observe the G and D band signal. The G-band $\left(\sim 1582 \mathrm{~cm}^{-1}\right)$ is the primary Raman active mode in graphite, and it provides evidence of the $\mathrm{sp}^{2}$ bonded carbon existing in planar sheet configurations. Thus, the $\mathrm{G}$ band signal indicates that the sample can be graphite, graphene, or CNT since all of them have the $\mathrm{sp}^{2}$ bonded carbon in the benzene structure. The D-band $\left(\sim 1350 \mathrm{~cm}^{-1}\right)$, known as the disorder or defect mode, originates from the edge configurations in graphene where the planar sheet configuration is disrupted. The mode is exhibited on the edge of graphene sheet or the open end of a carbon nanotube, where defects exist.

Fig. 6 and 7 show the data of measurements, including the reflectance $(R)$, the transmittance $(T)$, and the absorption $(A)$. The detailed measurement drawing can be referred from our past publication. ${ }^{30}$ Fig. 6 shows the measured results of EPDCNT films on a $100 \mathrm{~nm}$ Ta layer. The broadband spectral absorption is quite high in the range of $\lambda=300-2600 \mathrm{~nm}$ for $t_{\mathrm{CNT}}=4.5 \mu \mathrm{m}$ and $2.4 \mu \mathrm{m}$. Although the resonant cavity has not been formed, the Ta back reflection layer and the random distribution nature of EPD-CNTs have already effectively increased the absorption. The averaged absorption $\left(A_{\text {avg }}\right)$ is 0.97 for EPD-CNTs $\left(t_{\mathrm{CNT}}=4.5 \mu \mathrm{m}\right)$ with a Ta bottom layer in Fig. 6(a). A gradual decrease in absorption is observed with a decreased $t_{\mathrm{CNT}}$, and $\sim 0.8$ absorption is observed for the case of $t_{\mathrm{CNT}}=$ $0.7 \mu \mathrm{m}$ in Fig. 6(d). Fig. 7 shows the spectral absorption for CNTs with cavity design consisting of a Ta bottom layer and 3pair $\mathrm{Ti} / \mathrm{SiO}_{2}$ top layers. Firstly, Fig. 7 (b) depicts the UV-VIS-NIR 
(a)

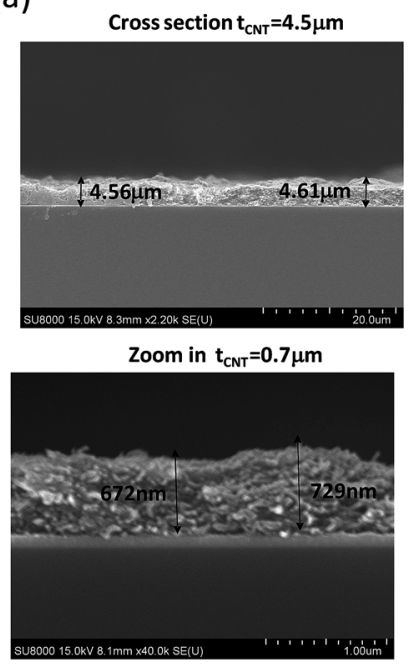

Cross section $\mathrm{t}_{\mathrm{CNT}}=0.7 \mu \mathrm{m}$

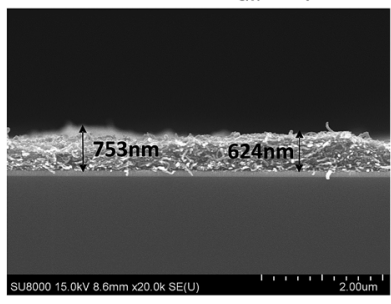

Top view

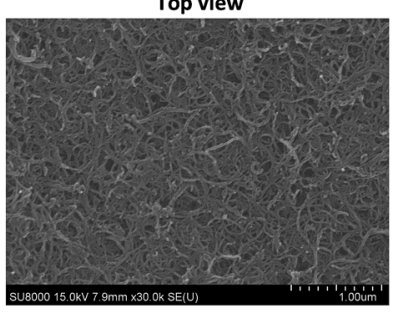

(b)

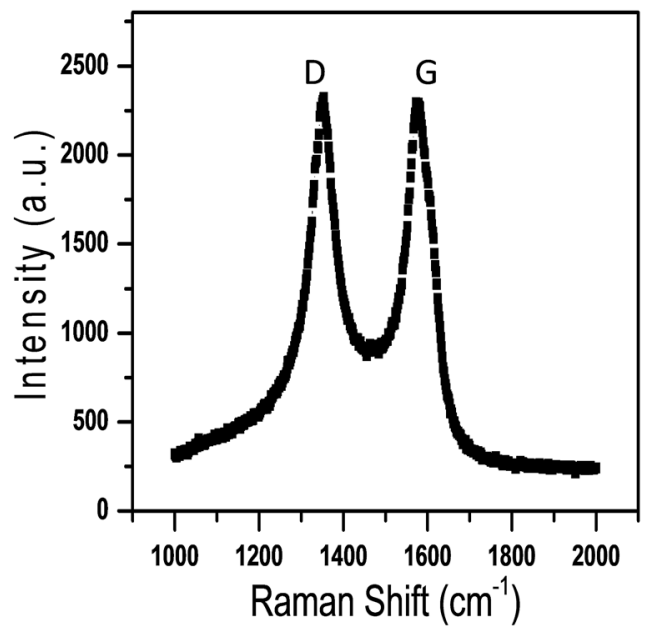

Fig. 5 (a) SEM micrographs of the EPD-CNT films in this experiment. The CNT films are deposited on a Ta bottom layer with various thickness. (b) Raman spectroscopy of the EPD-CNT film.

absorption spectrum from $\lambda=300 \mathrm{~nm}$ to $\lambda=2600 \mathrm{~nm}$. Fig. 7 (c) further shows the FTIR result of $1-R_{\mathrm{spec}}-T_{\mathrm{spec}}$ from $\lambda=1.2 \mu \mathrm{m}$ to $\lambda=10 \mu \mathrm{m}$. Due to the planar geometry, $1-R_{\mathrm{spec}}-$ $T_{\text {spec }} \cong 1-R-T=A$ (absorption). This point will be explained in more details in the method section. It can be extracted from Fig. 7 (c) that with the cavity design and $t_{\mathrm{CNT}} \sim 500 \mathrm{~nm}$, the high absorption exists from $\lambda=300 \mathrm{~nm}$ to $\lambda=4810 \mathrm{~nm}$. After $\lambda=5 \mu \mathrm{m}$, the spectral absorption begins to decay due to the geometry of (a)

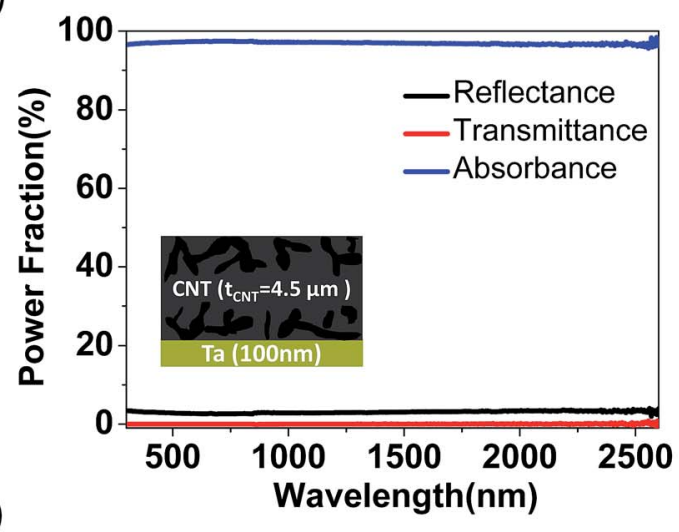

(c)

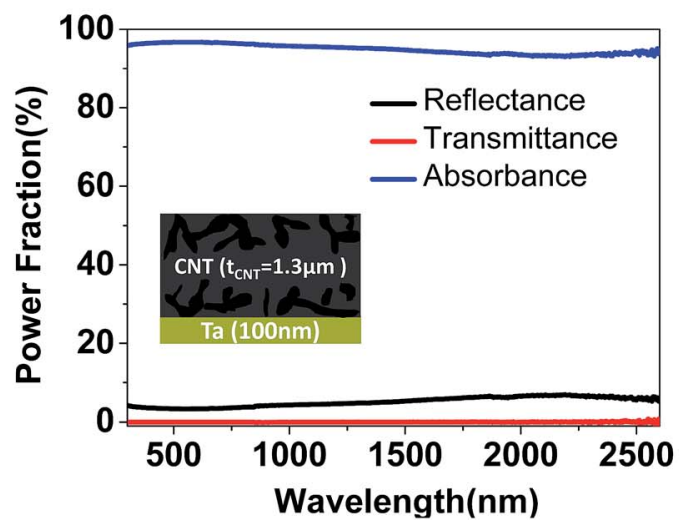

(b)
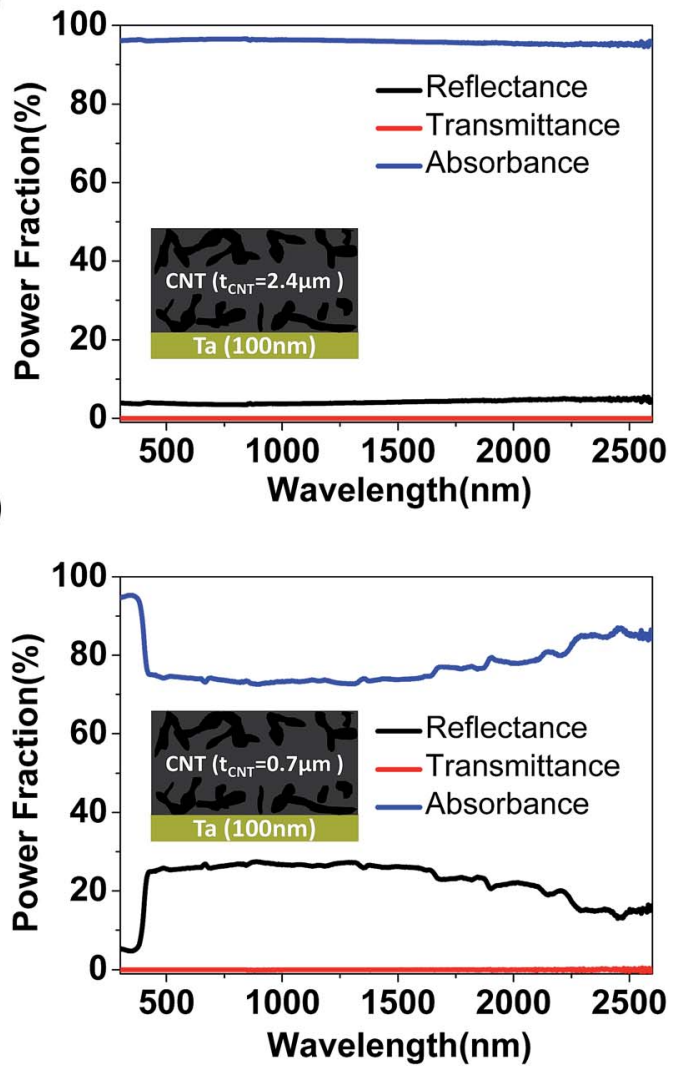

Fig. 6 The spectral transmittance $(T)$, reflectance $(R)$, absorption $(A)$ of the EPD-CNT layers of varying thicknesses on a $100 \mathrm{~nm}$ Ta bottom plate. The EPD-CNT layer thickness $\left(t_{\mathrm{CNT}}\right)$ is (a) $4.5 \mu \mathrm{m}$ (b) $2.4 \mu \mathrm{m}$ (c) $1.3 \mu \mathrm{m}$ (d) $0.7 \mu \mathrm{m}$, respectively. 
(a)

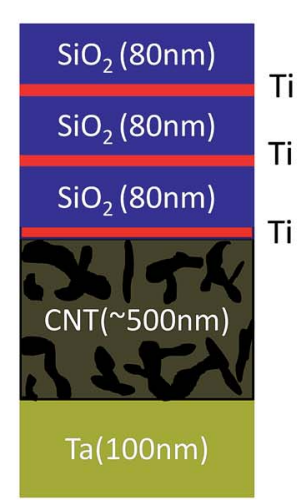

(b)

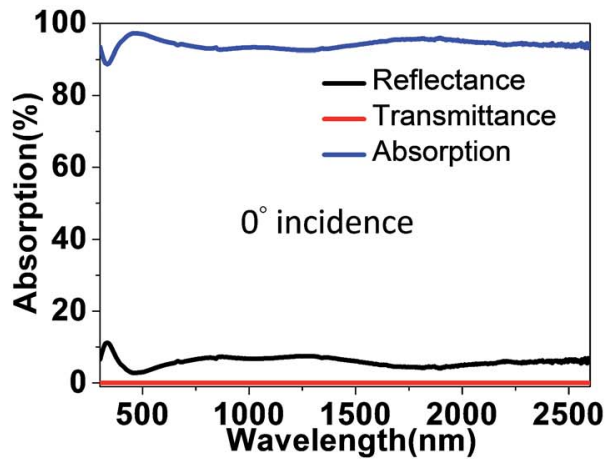

(c)

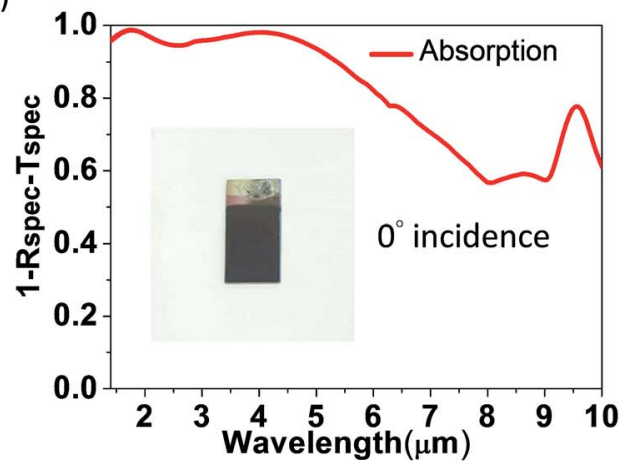

Fig. 7 The spectral absorption of cavity type CNTs with $100 \mathrm{~nm}$-Ta and 3 -pair $3 \mathrm{~nm}$-Ti/80 nm-SiO 2 cladding. $t_{\mathrm{CNT}} \sim 500 \mathrm{~nm}$. (a) EPD-CNT absorber layer specifications. (b) The UV-VIS-NIR absorption for $\lambda=300 \mathrm{~nm}$ to $\lambda=2600 \mathrm{~nm}$ (c) the FTIR spectrum from $\lambda=1.2 \mu \mathrm{m}$ to $\lambda=10 \mu \mathrm{m}$, the photograph of the cavity type EPD-CNT sample (inset). The specular reflectance $\left(R_{\text {spec }}\right)$ and specular transmittance $\left(T_{\text {spec }}\right)$ are measured. Since the structure is planar and the Bruker FTIR has numerical aperture NA $=\sin 10^{\circ}$, the $1-R_{\text {spec }}-T_{\text {spec }} \cong 1-R-T=A$.

$t_{\mathrm{CNT}} \sim 500 \mathrm{~nm}$ and $t_{\mathrm{SiO}_{2}}=80 \mathrm{~nm}$ for this experiment. To realize devices with a larger bandwidth or operation in longer wavelength regimes, increasing $t_{\mathrm{CNT}}$ and $t_{\mathrm{SiO}_{2}}$ can certainly be effective. It is worth to mention that the absorption and bandwidth in the experiment are further improved as compared to the simulation results mentioned in the previous section. The reason is that the CNTs in the simulation are assumed to be vertically aligned with refractive index $1.02+0.04 i,{ }^{22}$ but the CNTs deposited by EPD are randomly distributed. These entangled EPD-CNTs increase the probability of random light scattering in the CNT films, and thus absorption can be increased accordingly. This point has been evident from many solar cell light trapping literature. ${ }^{31-37}$ In short, the experimental results verify the proposed design, i.e., we can reduce the thickness of CNT absorbers by incorporating light scattering phenomenon in EPD-CNTs and a resonant cavity design using top $\mathrm{Ti} / \mathrm{SiO}_{2}$ layers and the bottom Ta layer.

Further material analysis is conducted to investigate the device structure and layer deposition in the cavity type CNTs with $100 \mathrm{~nm}$-Ta and 3-pair $3 \mathrm{~nm}$-Ti/ $80 \mathrm{~nm}-\mathrm{SiO}_{2}$ cladding. Fig. 8 demonstrates the STEM cross-section image of the fabricated device. The thickness of the EPD-CNT layer, in this case, is (a)
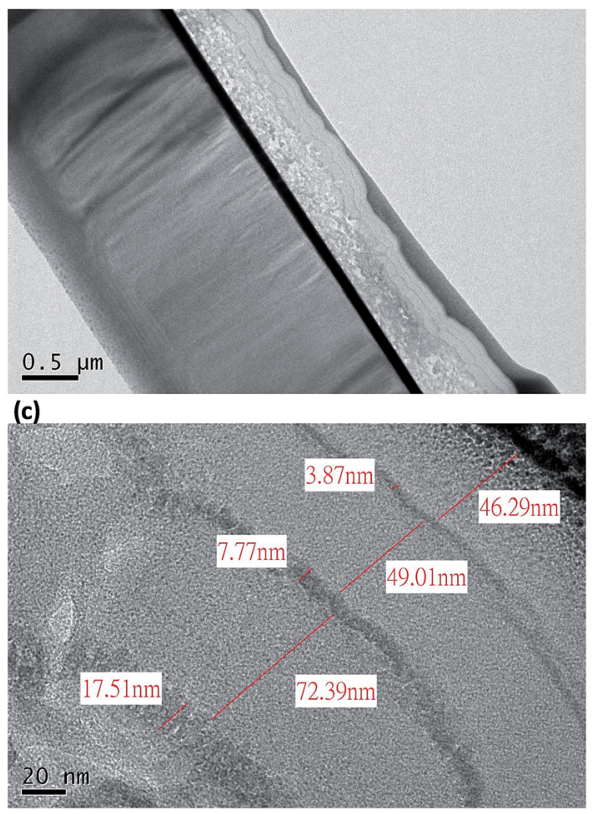

(b)
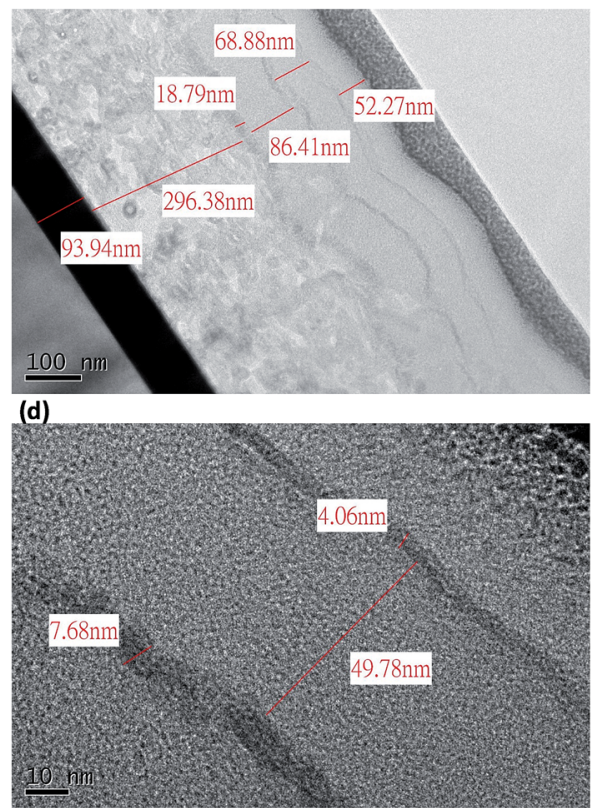

Fig. 8 Scanning transmission electron microscope (STEM) images of the EPD-CNT absorber with a $100 \mathrm{~nm}$ Ta bottom layer, a $~ 500 \mathrm{~nm}$ EPDCNT film, and 3 -pair $3 \mathrm{~nm}-\mathrm{Ti} / 80 \mathrm{~nm}-\mathrm{SiO}_{2}$ layers on top. The Ti layer suffers from diffusion especially for the first Ti layer that is sitting directly on the porous EPD-CNT film. Nevertheless, since the dimension of the Ti layer after diffusion is still much smaller than photon wavelength $(\lambda=300-2600 \mathrm{~nm})$, the effect of Ti diffusion on optical properties is not very pronounced. (a) The entire device structure. (b) The zoom-in image of the entire device structure. (c) and (d) The zoom-in images of the $\mathrm{Ti}_{\mathrm{i}} \mathrm{SiO}_{2}$ alternating layers. 
$\sim 500 \mathrm{~nm}$. The thickness variation is minimized by using a centrifuge to uniformly disperse the CNTs in the mixture of nitric and sulphuric acids $\left(\mathrm{HNO}_{3}\right.$ and $\left.\mathrm{H}_{2} \mathrm{SO}_{4}\right)$ solution although some layer thickness variation is still observed here. The thickness variation in CNT films is due to the EPD solution process, and the roughness and porosity of the CNT film can affect the morphology, uniformity, and the porosity of the subsequent $\mathrm{SiO}_{2}$ and Ti films. Further reduction in surface roughness and morphology is desired but it should be emphasized that the low-temperature chemical solution processing inevitably leads to surface morphology not comparable to vacuum deposition, and the surface roughness of the EPDCNT films here is comparable to other EPD CNT works in literature. ${ }^{38}$ In Fig. 8(c) and (d), it is observed that although asdeposited thickness of Ti thin film is $\sim 3 \mathrm{~nm}$, diffusion of Ti into $\mathrm{SiO}_{2}$ or CNT inevitably exists. This is due to the fact that the EPD-CNT film is highly texturized and porous with thickness non-uniformity as evident from Fig. 5, and this makes the $\mathrm{SiO}_{2}$ layer above less dense. From optics viewpoint, the slight mix-up of $\mathrm{Ti}$ and $\mathrm{SiO}_{2}$ layer is not detrimental as long as the dimension of the diffused Ti film is still much smaller than the photon wavelength. This is the essence of effective index theory in optics..$^{39,40}$

\section{Conclusions}

In this work, we propose an ultra-compact ultra-broadband ideal blackbody composed of a Ta bottom layer, EPD-CNTs, and alternating $\mathrm{Ti} / \mathrm{SiO}_{2}$ layers. In the past, due to the hollowcylindrical-shell structure of CNTs, the light coupling into the CNTs is extremely simple, similar to other porous materials. However, owing to the low material density and volume ratio, the thickness of most CNT perfect blackbody in literature is usually in the range of 10-100 $\mu \mathrm{m}$ (ref. 21-26) to achieve high absorption. As a result, although the high absorption property is fulfilled, the large thickness of CNT obstructs the integration of CNTs with other miniaturized optical devices. Here, we propose a thin CNT perfect absorber using EPD-CNTs with resonant cavity design consisting of alternating metal-dielectric layers. The simulation reveals that the resonances can be supported by the proposed structure where the EPD-CNT film is sandwiched between $\mathrm{Ti} / \mathrm{SiO}_{2}$ multilayers and the bottom Ta reflector. From the experimental results, it is evident that the proposed cavitytype EPD-CNT structure can indeed achieve high absorption from $\lambda=300 \mathrm{~nm}$ to $\lambda=2600 \mathrm{~nm}$ while the thickness of the EPDCNT layer is only $\sim 500 \mathrm{~nm}$, and the total device thickness is $<1 \mu \mathrm{m}$. The broadband high absorption of thin EPD-CNT absorbers is attributed to the random orientation of the EPDCNTs during EPD, which enhances light scattering and absorption. This aspect is quite different from many CNT perfect blackbodies in literature where vertically aligned CNTs are frequently used. ${ }^{21-26}$ In addition, the proper cavity design leads to enhanced photon confinement over a very broad bandwidth. We believe that this proposal constitutes the most promising CNT blackbody design due to a wide band absorption that could be achieved by employing a thin CNT layer. Also, the miniaturization of the CNT thickness and low-temperature processing enables its integration with other optical devices and makes it possible for CNTs to be categorized as a metamaterial perfect absorber (MPA) with dimension comparable to photon wavelengths.

\section{Methods}

\section{Sample fabrication}

The method we used to fabricate the carbon nanotubes (CNTs) film is electrophoretic deposition (EPD). The EPD is an economical method that only requires simple equipment and can be completed at low temperature $\left(<120^{\circ}\right)$, and thus it is commonly employed in the fabrication of films from colloidal suspensions. The EPD is essentially composed of two steps. The first step is suspending CNTs in a liquid and applying a voltage to force CNTs moving toward the electrode. In the second step, the nanoparticles coagulate at the electrode and form a coherent coating of CNTs. Initially, we disperse $1 \mathrm{~g}$ CNTs in a $40 \mathrm{~mL}$ mixture of nitric and sulphuric acids $\left(\mathrm{HNO}_{3}\right.$ and $\mathrm{H}_{2} \mathrm{SO}_{4}$ ). The volumetric proportion of nitric acids to sulphuric acids is $1: 3$. Therefore, the volume of the nitric acid is $10 \mathrm{~mL}$, and the volume of the sulphuric acid is $30 \mathrm{~mL}$. The purpose of this step is to purify and to disperse the as-produced CNTs since they are usually entangled or aggregated and are full of impurities. Afterward, we heat up the solution on a hot plate at $120^{\circ} \mathrm{C}$ for 30 minutes, and the solution is rinsed with deionized water until the $\mathrm{pH}$ value is $\sim 7$. The excess water is then evaporated in an oven at $80{ }^{\circ} \mathrm{C}$ for ten hours. After purification, we can acquire $0.5 \mathrm{~g}$ CNTs. i.e., the production is $\sim 50 \%$. Subsequently, we triturate $0.03 \mathrm{~g}$ after-processing CNTs and pour the powders into $60 \mathrm{~mL}$ isopropyl alcohol and $0.4 \mathrm{~mL} \mathrm{HCl}$ to prepare the CNT suspensions for EPD. The CNT suspensions are sonicated in an ultrasonic bath for 2 hours to have well-dispersed suspensions before EPD. Ta/Si is used as the cathode during EPD, and we have deposited $100 \mathrm{~nm}$ Ta on a silicon wafer previously using an ULVAC $^{\text {TM }}$ ENTRON W200 sputter. The anode is stainless steel. Next, we apply an electric field between these two electrodes, and the nanotubes will aggregate at the cathode to form a CNT film. The CNT film is then baked at $60^{\circ} \mathrm{C}$ in oven for 5 minutes. After the deposition of CNTs has been finished, we deposit Ti/ $\mathrm{SiO}_{2}$ alternating layers by electron-gun (e-gun) evaporator AST PEVA 600I. The pressure is kept $<3 \times 10^{-6}$ torr for Ti deposition and $<5 \times 10^{-6}$ torr for $\mathrm{SiO}_{2}$ deposition. We deposit $\sim 3 \mathrm{~nm}$ Ti on the top of CNTs and then deposit $80 \mathrm{~nm} \mathrm{SiO}_{2}$ on Ti. The process is repeated until three pairs of alternating layers have been fabricated. The EPD-CNT film is highly texturized and porous, and thus makes the $80 \mathrm{~nm} \mathrm{SiO}_{2}$ layer above less dense. The asdeposited thickness of Ti thin film is $\sim 3 \mathrm{~nm}$, but because Ti is deposited on the less dense $\mathrm{SiO}_{2}$ layers or the porous CNT film, some diffusion of $\mathrm{Ti}$ into $\mathrm{SiO}_{2}$ or CNT inevitably exists.

\section{Sample measurements}

In this work, the reflectance $(R)$, transmittance $(T)$, and absorption $(A)$ refer to total reflectance, total transmittance, and total absorption. Thus, in some places, the term "total" is not written out explicitly for succinct labeling. On the other hand, 


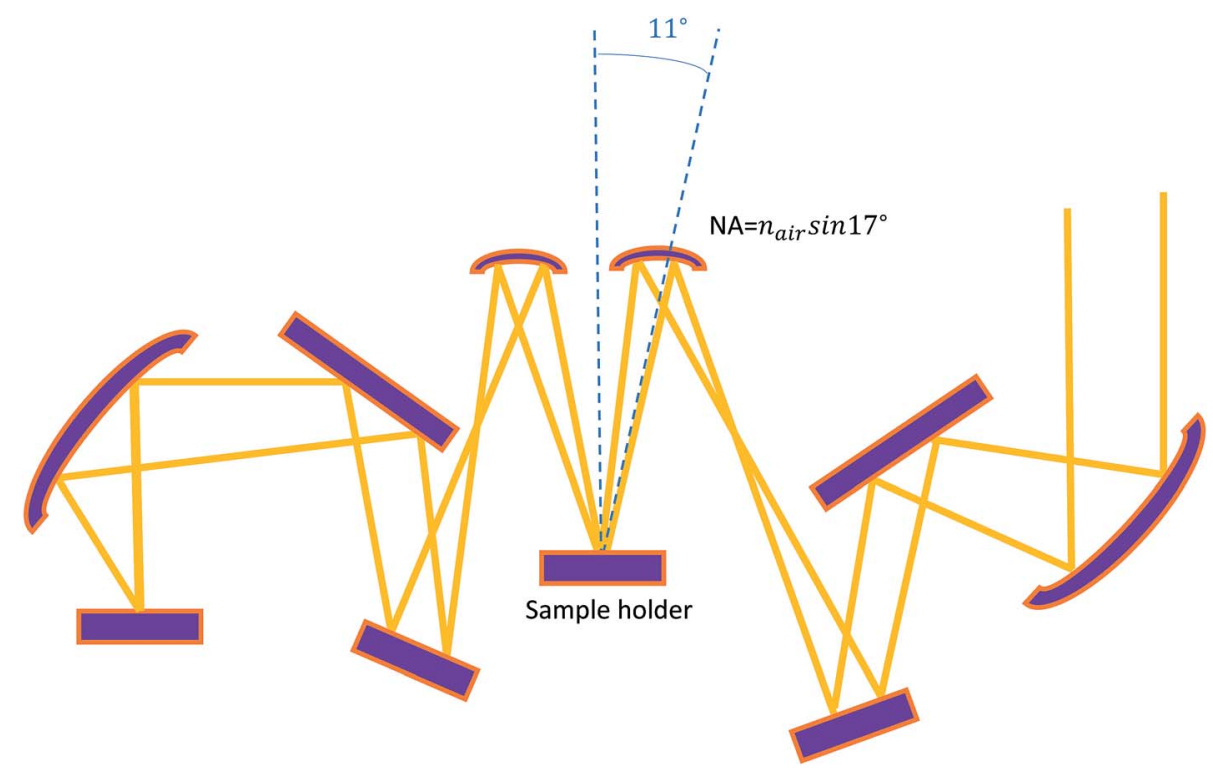

Fig. 9 Illustration of the FTIR measurement set-up in Bruker IFS 66v/S.

specular reflectance $\left(R_{\text {spec }}\right)$ and specular transmittance $\left(T_{\text {spec }}\right)$ means the optical power that can be collected by the numerical aperture (NA) of the lens/mirrors set of the equipment in the case where no integration sphere is present. Normally $R_{\mathrm{spec}}$ and $T_{\text {spec }}$ are the power enclosed in a solid angle around the reflection line where the solid angle is determined by the numerical aperture (NA).

The measurement of UV-VIS-NIR absorption spectrum is by measuring the reflectance $(R)$ and the transmittance $(T)$. The absorption $(A)$ is then calculated by $A=1-R-T$. The integration sphere collects all diffracted power so the total reflectance $(R)$, total transmittance $(T)$, and total absorption $(A)$ can be measured at normal incidence. Hitachi U-4100 is used where the light source is a quickly mountable type deuterium lamp for the ultraviolet region and a $50 \mathrm{~W}$ halogen lamp for the visiblenear-infrared region. The pre-monochromator is Littrow monochromator using a diffraction grating, and the main monochromator is also of the grating type with two switchable diffraction gratings. The detector is photomultiplier for the UVVIS region and a cooling type PbS photoconductive detector for the NIR region.

The Fourier transform infrared (FTIR) spectroscopy is used for $\lambda=1.2 \mu \mathrm{m}$ to $\lambda=10 \mu \mathrm{m}$. Bruker IFS $66 \mathrm{v} / \mathrm{S}$ Fourier transform infrared spectroscopy (FTIR) is used. The resolution is $0.25 \mathrm{~cm}^{-1}$. The light source is silicon carbide (SiC) glow bar, and the detector is a pyroelectric detector using deuterated lanthanum $\alpha$ alanine doped triglycine sulphate (DLATGS) with $\mathrm{KBr}$ window. In our FTIR measurement using Bruker machine, the incidence angle is the nearly-normal direction at 11 degrees $\left(11^{\circ}\right)$. Using e-gun evaporation or other low-cost deposition techniques such as sputtering, solution processes, and inkjet printing, is promising for MWIR/LWIR perfect absorbers because the surface roughness is a less severe concern for MWIR/LWIR regime. This is due to the large MWIR/LWIR wavelength dimension compared to the vertical/lateral sample surface roughness in the range of nanometers. As a result, the incident photons essentially cannot perceive the surface texture of the films. The fact of no need for MBE or ALD in the planar MWIR/LWIR perfect absorber structure here enhances its practicability for MWIR/LWIR applications. The setup for the FTIR measurement is shown in Fig. 9. In most FTIR machines there is not integration sphere, due to the long wavelength radiation is more robust to surface roughness. Since there is no integration sphere in FTIR machine, $R_{\text {spec }}$ and $T_{\text {spec }}$ are measured. Due to the fact that our structure is a planar structure and the fact that the Bruker ${ }^{\mathrm{TM}}$ FTIR has NA $=\sin 15^{\circ}, R_{\mathrm{spec}}$ and $T_{\text {spec }}$ are close to $R$ and $T$, and thus $1-R_{\text {spec }}-T_{\text {spec }} \cong 1-R$ $-T=A$. We have verified that the diffraction and light scattering from a $700 \mathrm{~nm}$ EPD CNT film are fully within $10^{\circ}$ for UV$\operatorname{VIS}-\mathrm{NIR}(\lambda=300-2600 \mathrm{~nm})$, and this means the $1-R_{\text {spec }}-T_{\text {spec }}$ is exactly the same as $1-R-T=A$ for this wavelength regime in our FTIR measurement. Further increase in wavelength is unlikely to increase the light scattering angle due to the fact that the surface profiling and thickness variation is $<50 \mathrm{~nm}$ from the SEM micrographs shown previously, and this dimension is insufficient to cause significant far-field light diffraction or scattering for wavelengths beyond UV-VIS-IR. As a result, the value of $1-R_{\text {spec }}-T_{\text {spec }}$ can be used to infer the absorption behaviour of the sample in our case.

\section{Conflicts of interest}

The authors declare no competing financial interests.

\section{Acknowledgements}

We acknowledge the support for this work from the Ministry of Science and Technology (MOST), Taiwan under grant number MOST 104-2221-E-009-115-MY2. 


\section{References}

1 N. Liu, M. Mesch, T. Weiss, M. Hentschel and H. Giessen, Nano Lett., 2010, 10, 2342-2348.

2 H.-T. Chen, W. J. Padilla, M. J. Cich, A. K. Azad, R. D. Averitt and A. J. Taylor, Nat. Photonics, 2009, 3, 148-151.

3 D. Dregely, R. Taubert, J. Dorfmüller, R. Vogelgesang, K. Kern and H. Giessen, Nat. Commun., 2010, 2, 267.

4 X. Liu, T. Tyler, T. Starr, A. F. Starr, N. M. Jokerst and W. J. Padilla, Phys. Rev. Lett., 2011, 107, 045901.

5 C. Wu, B. Neuner, J. John, A. Milder, B. Zollars, S. Savoy and G. Shvets, J. Opt., 2012, 14, 024005.

6 C. M. Watts, X. Liu and W. J. Padilla, Adv. Opt. Mater., 2012, 24, OP98-OP120.

7 H. Tao, N. I. Landy, C. M. Bingham, X. Zhang, R. D. Averitt and W. J. Padilla, Opt. Express, 2008, 16, 7181-7188.

8 X. Liu, T. Starr, A. F. Starr and W. J. Padilla, Phys. Rev. Lett., 2010, 104, 207403.

9 Y. Avitzour, Y. A. Urzhumov and G. Shvets, Phys. Rev. B: Condens. Matter Mater. Phys., 2008, 79, 045131.

10 K. Aydin, V. E. Ferry, R. M. Briggs and H. A. Atwater, Nat. Commun., 2011, 2, 517.

11 H. Tao, C. M. Bingham, A. C. Strikwerda, D. Pilon, D. Shrekenhamer, N. I. Landy, K. Fan, X. Zhang, W. J. Padilla and R. D. Averitt, Phys. Rev. B: Condens. Matter Mater. Phys., 2008, 78, 241103.

12 H. Tao, C. M. Bingham, D. Pilon, K. Fan, A. C. Strikwerda, D. Shrekenhamer, W. J. Padilla, X. Zhang and R. D. Averitt, J. Phys. D: Appl. Phys., 2010, 43, 225102.

13 J. Hao, J. Wang, X. Liu, W. J. Padilla, L. Zhou and M. Qiu, Appl. Phys. Lett., 2010, 96, 251104.

14 Y. Cui, K. H. Fung, J. Xu, H. Ma, Y. Jin, S. He and N. X. Fang, Nano Lett., 2012, 12, 1443-1447.

15 D. Ji, H. Song, X. Zeng, H. Hu, K. Liu, N. Zhang and Q. Gan, Sci. Rep., 2014, 4, 4498.

16 C. Argyropoulos, K. Q. Le, N. Mattiucci, G. D'Aguanno and A. Alu, Phys. Rev. B: Condens. Matter Mater. Phys., 2013, 87, 205112.

17 Y. K. Zhong, S. M. Fu, N. P. Ju, M. Tu, B. Chen and A. Lin, IEEE Photonics J., 2016, 8, 2200109.

18 M. Farhat, T.-C. Cheng, K. Q. Le, M. M.-C. Cheng, H. Bagci and P.-Y. Chen, Sci. Rep., 2016, 6, 19984.

19 E. Dervishi, A. R. Biris, J. A. Driver, F. Watanabe, S. Bourdo and A. S. Biris, J. Catal., 2013, 299, 307-315.
20 Y. Ra'di, C. R. Simovski and S. A. Tretyakov, Phys. Rev. Appl., 2015, 3, 037001.

21 A. Lenert, D. M. Bierman, Y. Nam, W. R. Chan, I. Celanovic, M. Soljacic and E. N. Wang, Nat. Nanotechnol., 2014, 9, 126130.

22 H. Shi, J. G. Ok, H. W. Baac and L. J. Guo, Appl. Phys. Lett., 2011, 99, 211103.

23 K. Mizuno, J. Ishii, H. Kishida, Y. Hayamizu, S. Yasuda, D. N. Futaba, M. Yumura and K. Hata, Proc. Natl. Acad. Sci. U. S. A., 2009, 106, 6044-6047.

24 F. J. García-Vidal, J. M. Pitarke and J. B. Pendry, Phys. Rev. Lett., 1997, 78, 4289-4292.

25 A. Cao, X. Zhang, C. Xu, B. Wei and D. Wu, Sol. Energy Mater. Sol. Cells, 2002, 70, 481-486.

26 Z.-P. Yang, L. Ci, J. A. Bur, S.-Y. Lin and P. M. Ajayan, Nano Lett., 2008, 8, 446-451.

27 N. Selvakumar, S. B. Krupanidhi and H. C. Barshilia, Adv. Mater., 2014, 26, 2552-2557.

28 A. R. Boccaccini, J. Cho, J. A. Roether, B. J. C. Thomas, E. Jane Minay and M. S. P. Shaffer, Carbon, 2006, 44, 3149-3160.

29 Rsoft, Rsoft CAD User Manual, Rsoft Design Group, New York, 8.2 edn, 2010.

30 Y. K. Zhong, Y.-C. Lai, M.-H. Tu, B.-R. Chen, S. M. Fu, P. Yu and A. Lin, Opt. Express, 2016, 24, A832-A845.

31 F. Pratesi, M. Burresi, F. Riboli, K. Vynck and D. S. Wiersma, Opt. Express, 2013, 21, A460-A468.

32 E. R. Martins, J. Li, Y. Liu, V. Depauw, Z. Chen, J. Zhou and T. F. Krauss, Nat. Commun., 2013, 4, 2665.

33 M. Burresi, F. Pratesi, K. Vynck, M. Prasciolu, M. Tormen and D. S. Wiersma, Opt. Express, 2013, 21, A268.

34 A. Bozzola, M. Liscidini and L. C. Andreani, Prog. Photovoltaics Res. Appl., 2014, 22(12), 1237-1245.

35 X. Sheng, S. G. Johnson, J. Michel and L. C. Kimerling, Opt. Express, 2011, 19, A841-A850.

36 C. Lin and M. L. Povinelli, Opt. Express, 2011, 19, A1148A1154.

37 A. Lin and J. D. Phillips, Sol. Energy Mater. Sol. Cells, 2008, 92, 1689-1696.

38 B. J. C. Thomas, A. R. Boccaccini and M. S. P. Shaffer, J. Am. Ceram. Soc., 2005, 88, 980-982.

39 S. L. Chuang, Physics of Photonic Devices (Wiley Series in Pure and Applied Optics), Wiley, New York, 2nd edn, 2009.

40 P. Bhattacharya, Semiconductor Optoelectronic Devices, 2nd edn, Prentice-Hall, Upper Saddle River, NJ, 2006. 